\title{
A critical Appraisal of Heterogeneity in Obsessive-Compulsive Disorder Using Symptom-Based Clustering Analysis
}

Hesam Hasanpour ${ }^{\mathrm{a}}$, Sareh Asadi ${ }^{\mathrm{b}}$, Ramak Ghavamizadeh Meibodi, Azin Daraeian ${ }^{\mathrm{c}}$, Abolhassan Ahmadiani $^{\mathrm{c}}$, Jamal Shams ${ }^{\mathrm{d}^{*}}$, Keivan Navi $^{\mathrm{a}^{*}}$,

a. Department of Electrical and Computer Engineering, Shahid Beheshti University, Tehran, Iran

b. NeuroBiology Research Center, Shahid Beheshti University of Medical Sciences, Tehran, Iran

c. Neuroscience Research Center, Shahid Beheshti University of Medical Sciences, Tehran, Iran

d. Behavioral Sciences Research Center, Shahid Beheshti University of Medical Sciences, Tehran, Iran

*Corresponding authors:

Dr Jamal Shams, Behavioral Sciences Research Center, Imam Hossain Educational Hospital, Shahid Beheshti University of Medical Sciences, Madani Ave, P.O. Box 1617763141, Tehran, Iran

Tel: + 98-21-77553074 Fax: + 98-21-77553074, E-mail: $\underline{\text { J shams@sbmu.ac.ir }}$

Prof. Keivan Navi, Department of Electrical and Computer Engineering, Shahid Beheshti University, GC, Tehran, Iran

Tel+ 98-21-29904195 Email:navi@sbu.ac.ir

(C) 2016. This manuscript version is made available under the Elsevier user license http://www.elsevier.com/open-access/userlicense/1.0/ 


\begin{abstract}
Obsessive-compulsive disorder (OCD) encompasses a broad range of symptoms and is commonly considered a heterogeneous condition. Attempts were made to define discrete OCD subtypes using a range of symptom-based methods including factor and cluster analyses. The present study aims to find the most appropriate clustering model based on Yale-Brown obsessive-compulsive scale (YBOCS) checklist explaining OCD heterogeneity.

Five different clustering algorithms (FCM, K-means, Ward, Ward + K-means and Complete) applied on YBOCS symptoms of 216 patients with OCD. Data studied as four different sets including item-level raw data, item-based factor scores, category-level raw data and categorybased factor scores and clustering results for 2 to 6 cluster solutions evaluated by four clustering indices (Davies-Bouldin, Calinski-Harabasz, Silhouettes and Dunn indices).

Two-cluster solution was detected as the most appropriate model for item and category-based clustering analyses of YBOCS checklist symptoms. Patients in each cluster were characterized based on their clinical and demographic properties and results showed that they had similar patterns of symptoms but in different severities.

Heterogenity of OCD based on the YBOCS-symptoms has been challenged as OCD patients were classified based on their symptom severity not their symptom patterns. More investigations need to find appropriate measures explaining OCD heterogeneity with clinical importance.
\end{abstract}

Keyword: Clustering algorithms; OCD heterogeneity; OCD Subtypes; OCD symptoms; YBOCS 


\section{Introduction}

Obsessive-compulsive disorder (OCD) is a neuropsychiatric heterogeneous condition that affects $1-3 \%$ of the population worldwide (Hasler et al., 2005). OCD patients may display different symptom patterns and illness courses. This phenotypic variability has led to the hypothesis that OCD is a heterogeneous disorder (Lochner and Stein, 2003; Mataix-Cols et al., 2005). Various subtypes of OCD have been reported in the literature obtained from different analytic approaches (Bloch et al., 2008). Different OCD subtypes may have different etiologic pathways and justify variability in genetic, neural, and neuropsychological correlates.(McKay et al., 2004). A better understanding of OCD clinical subtypes may lead to advances in understanding the psychobiology of the disorder, and improved treatments.

Researchers have used several different measures for identifying OCD subtypes including obsessive and compulsive symptom patterns (Calamari et al., 1999; Lochner C, 2008), age at onset of OCD (Noshirvani et al., 1991), differential treatment response, genetic backgrounds of individuals diagnosed with OCD (Katerberg et al., 2010) and the presence of comorbid conditions (Mataix-Cols et al., 2000).

The most frequently used strategy for identifying subtypes of OCD has involved the evaluation of obsessive and compulsive symptom commonalities in patients (Calamari et al., 1999). The most comprehensive and widely used inventory available and the source of data for investigation of OCD symptoms and severity is the Yale Brown Obsessive Compulsive Scale (YBOCS) (Goodman et al., 1989). YBOCS symptom checklist (YBOCS-CL) classifies the obsessions and compulsions in 13 specific categories and 2 
miscellaneous groups. The item composition of each of the checklist categories was derived through clinical judgment to group the symptoms into clinically coherent categories, and to create a checklist that would facilitate data collection (Feinstein et al., 2003).

There are two common types of methods for analysis of OCD data: variable-centered factor analysis and person-centered clustering (Calamari et al., 2004; Delucchi et al., 2011; Katerberg et al., 2010). Factor analysis is used for investigating the relationships of variables for complex concepts that are not easily measured directly. This method reduces a large number of variables into a few interpretable underlying factors. Category and item based factor analyses of (YBOCS-CL) in different studies resulted into four or five factor models (Bloch et al., 2008).

Clustering strategies and latent class analysis (LCA) are person-centered methods that are used for identifying OCD symptom-specific subtypes (Calamari et al., 1999; Calamari et al., 2004; Delucchi et al., 2011; Nestadt et al., 2003; Nestadt et al., 2009). In cluster analysis, subjects are grouped into several clusters by maximizing between group differences and minimizing within group variation on the chosen set of criteria while LCA assumes an underlying latent class membership among subjects that gives rise to the subject subgroups (Vermunt and Magidson, 2004). In factor analysis, subjects may have loadings on all the identified factors so it will be hard to relate each person to a specific dimension. Cluster and latent class analyses enables researchers to form relatively homogenous subject groups within complex data set (Calamari et al., 1999). 
The first reported OCD cluster analysis OCD personality data were clustered by Ward's method and were verified by K-means clustering algorithm. Four clusters that were different with each other in response to behavioral therapy were identified (Fals-Stewart and Lucente, 1993). Calamari et al. used Ward's hierarchical agglomerative cluster analysis followed by K-means clustering of YBOCS-CL scores and identified five groups that were characterized by dominant symptom patterns (Calamari et al., 1999). Further validation of results by subgroup characteristics related to treatment response or the etiology of OCD showed greater support for a seven subgroup taxonomy (Calamari et al., 2004). Another study clustered OCD patients into 3 clusters based on their comorbidity of obsessive-compulsive spectrum disorders (OSCDs) using Ward's method (Lochner et al., 2005). Cluster analysis of 45 items of YBOCS-CL reported by this sample identified six-cluster solution (Lochner C, 2008).

Attempts for classifying OCD symptoms using LCA resulted in different models based on the symptoms types. LCA analysis of neurologic signs, electroencephalographic abnormalities, attention deficit, and developmental disorder to investigate birth complications and neurologic abnormalities in individuals with OCD identified a two class model, organic and nonorganic class suggesting that OCD belongs to nonorganic class and is not the result of organic brain disease (Thomsen and Jensen, 1991). LCA analysis of OCD using patterns of comorbidity detected 4 latent classes: a minimal disorders class; a recurrent major depression and generalized anxiety class; a highly comorbid class, consisting of individuals with multiple comorbid psychiatric disorders; and a tic disorder, panic, and agoraphobia class (Nestadt et al., 2003). The subsequent study using comorbidity and clinical characteristics such as sex, age at onset, and OCD 
symptom type resulted in a 2-class solution was characterized by lesser and greater comorbidity classes and a 3-class solution that consisted of an OCD-only class ( \pm major depression), an OCD + tics class, and an OCD + affective disorders (highly comorbid) class (Nestadt et al., 2009). Latent class analysis of the 8-item obsessive-compulsive scale of the Child Behavior Checklist suggested a 4-class model, including a "no symptoms" class, a "worries and has to be perfect" class, a "thought problems" class, and an "obsessive-compulsive scale items" class (Althoff et al., 2009). In another study analysis of YBOCS symptoms to detect latent classes yielded 3 classes differed only in frequency of symptom endorsement (Delucchi et al., 2011).

Different OCD symptom subtypes may have different psychopathological mechanisms requiring specific treatment strategies. The present study aims to shed more light on OCD symptom subtypes and suggest the best model for clustering OCD patients. We have used several clustering strategies to for categorizing OCD patients based on their YBOCS symptoms and compared the results with those of LCA.

\section{Method}

\subsection{Participants and clinical assessment}

Two hundred and sixteen patients with OCD ( $n=216,146$ female and 70 male), were included in the study (mean age at assessment, $33.7 \pm 10.2$ ) (Table 1).

Subjects were interviewed by an experienced clinician, and met the Diagnostic and Statistical Manual of Mental Disorders (DSM IV-TR) (Association, 1994) criteria for OCD on the Structured Clinical Interview for Axis I Disorders. The exclusion criteria were having a history of psychotic disorders or mental retardation, reporting severe neurological pathology and history of substance use, diagnosis with other DSM-IV-TR 
Axis I disorders except depression, anxiety or tic disorder. The socio-demographic data was applied through a questionnaire. The Persian version of YBOCS severity scale and checklist (Rajezi Esfahani et al., 2012) was used to assess the severity and types of current obsession and compulsion symptoms. The study was approved by the Research Ethics Committee of Neuroscience Research Center, Shahid Beheshti University of Medical Sciences (Project No. 492.1).

\subsection{Data analysis}

Scores on the YBOCS -CL were analyzed in two levels: item and category levels. In item-level analyses the 5 point Likert scale data without any conversion was used as raw data. For category-level analyses each category was scored 0,1 or 2 in the same manner as in several prior studies (Baer, 1994; Calamari et al., 1999). To increase comparability to previous studies (Calamari et al., 1999; Denys et al., 2004; Katerberg et al., 2010), the items of miscellaneous obsession and compulsion categories and 10 open-ended (i.e., 'other') items were excluded.

Exploratory factor analysis was conducted in both item and category levels as explained previously (Asadi et al., 2016). Briefly, exploratory factor analysis was conducted on YBOCS checklist data using the maximum likelihood estimation method, followed by varimax rotation. Results are summarized in five-factor model for item-based and fourfactor model for category-based data (Table 2).

Raw item-level and category-level data as well as factor scores at each level were subjected to cluster analysis. Two common types of clustering methods are partitional and hierarchical clustering. Partitional clustering such as K-means attempts to directly decompose the data set into a set of disjoint clusters (MacQueen, 1967). 
Since the result of K-means algorithm is sensitive to initial cluster centers (centroids), we used another strategy to calculate these centroids. In Ward+K-means algorithm, Ward's hierarchical agglomerative procedure was used to establish initial clusters followed by K-means clustering with the number of clusters and centroids determined based on the hierarchical solution.

Fuzzy C-means (FCM) was also used in the current study that is an extension of classical C-Means algorithm for fuzzy applications (Bezdek et al., 1984). Completelinkage clustering was also used to analyze data. In this method, distance between clusters equals the distance between two elements (one in each cluster) that are farthest away from each other (Defays, 1977).

To evaluate different clustering algorithms, four indices (Dunn index, Davies-Bouldin index, Calinski-Harabasz index and Silhouettes validity index) were used.

Dunn index is a metric of how well a set of clusters represent compact well-separated clusters (Ilc, 2012). The higher Dunn's value indicates better clustering. Davies-Bouldin index (Davies and Bouldin, 1979) and Calinski-Harabasz (Caliński and Harabasz, 1974) are internal evaluation measures. The Davies-Bouldin index measures the average of similarity between each cluster and its most similar one. The lower Davies-Bouldin index means better cluster configuration. Calinski-Harabasz index evaluates the cluster validity based on the average between and within cluster sum of squares. The maximal Calinski-Harabasz index value indicates the best clustering of the data. Silhouettes validity index (Rousseeuw, 1987) measures the distance between each data point, the centroid of the cluster it was assigned to and the closest centroid belonging to another 
cluster. The average Silhouette indicates weak clustering model if near zero, strong if near +1 and mis-clustering if near -1 .

To assess cluster differences based on demographic and clinical characteristics groups of patients separated by clustering algorithms were compared by T-test (continues variables) or Chi-square (categorical variables). All statistical analyses were conducted using Matlab version 2014a (The MathWorks Inc.).

\section{Results}

\subsection{Sample characteristics}

Demographic and clinical data were summarized in table 1. The total sample included 216 subjects $68 \%(n=146)$ of whom were female and $32 \%(n=70)$ were male. The majority of subjects were married (68\%), low educated ( $76 \%$ school dropout or diploma) and unemployed (55\%). Most of the patients reported the presence of the familial history of a psychiatric disorder (75\%). The mean ages ( \pm SD) of assessment and of onset were $33.7 \pm 10.2$ and $23.7 \pm 10.7$ years, respectively. The mean obsession, compulsion and total severity scores $( \pm S D)$ reported by patients were $10.3 \pm 4.7,8.9 \pm 5.9$ and $18.6 \pm 8.8$, respectively.

\subsection{Factor analyses}

Exploratory factor analyses at item level resulted in five factors (Table 2): 1. aggression obsessions and related checkings; 2 . contamination obsessions and cleaning compulsions; 3. hoarding obsession and compulsion, symmetry obsession, repeating, counting, ordering and arranging compulsions; 4 . sexual obsessions and 5. somatic obsessions and checking compulsion tied to somatic obsessions (Asadi et al., 2016). 
As presented in table 2 category-based exploratory factor analysis specified 4 factors including: 1. aggression, sexual, religious and somatic obsessions and checking and repeating compulsions; 2 . symmetry obsessions and cleaning, counting and ordering compulsions; 3. contamination obsessions and cleaning compulsions and 4. hoarding obsession and compulsion.

\subsection{Cluster analysis}

Five different algorithms (FCM, K-means, Ward, K-means+Ward and Complete) applied on four sets of data: 1 . item-level raw data; 2 . item-based factor scores; 3 . categorylevel raw data; 4. category-based factor scores. Cluster analyses were conducted for 2 to 6 cluster solutions and results were compared for 4 clustering indices (DaviesBouldin, Calinski-Harabasz, Silhouettes and Dunn indices) to find the best clustering model for OCD patients' data.

As shown in figure $1 \mathrm{~A}_{1}$ two-cluster solutions resulted from conducting all algorithms on item-level raw data had the least Davies-Bouldin value. Less Davies-Bouldin value shows better clustering solution. Similar results obtained from applying these five algorithms on the other data sets $\left(1 \mathrm{~A}_{2}-1 \mathrm{~A}_{4}\right)$ except the results of clustering item-based and category-based factor scores by Complete algorithm. In these two cases six-cluster models had the least Davies-Bouldin indices but the values were the same or even more than index values of two-cluster solutions resulted from the other algorithms.

For the other three indices (Calinski-Harabasz, Silhouettes and Dunn indices) higher index means more appropriate model. Applying almost all of the algorithms on four data sets detected 2-cluster models with the most index values (Fig 1: $B_{1}-B_{4}, C_{1}-C_{4}$ and $D_{1}$ - 
$D_{4}$ ). Again Complete algorithm showed different results (1B2, 1B4, 1C4, 1D3 and 1D4), however most of two-clustering models specified by other clustering algorithms owned higher indices than models resulted from Complete algorithm. Based on these results two-cluster solutions were chosen as the best clustering models for YBOCS-CL symptoms and were assessed further for the clinical and demographic characteristics.

As presented in figure 2, two clusters obtained from applying five clustering algorithms on four sets of data were compared based on the pattern and severity of obsessivecompulsive symptoms.

The patients separated into two groups by conducting clustering algorithms on itemlevel raw did not show significant differences in the patterns of symptoms but they had different severity $\left(\mathrm{Fig} 2 \mathrm{~A}_{1}\right)$. One group showed more severe symptoms than the other. Similar results obtained from clustering of category-level raw data $\left(\mathrm{Fig} 2 \mathrm{~B}_{1}\right)$. Clustering item-based (Fig $\left.2 \mathrm{~A}_{2}\right)$ and category-based $\left(\mathrm{Fig} 2 \mathrm{~B}_{2}\right)$ factor scores also separated subjects according to their mean severity of underlying factors.

For further clarification of clinical and demographic differences of clusters resulted from clustering of OCD data sets, different variables compared by Chi-square test (gender, marital status, educational level, occupation, familial history of psychiatric disorder, insight and avoidance levels) or T-test (age at assessment, age of onset, obsession, compulsion and depression scores) between two subgroups. Table 3 presents between subgroups differences of clustering item-level raw data and item-based factor scores. Almost no significant differences in demographic characteristics (gender, marital status, educational levels, occupation and familial history of psychiatric disorders) were detected between 2 clusters resulted from clustering item data sets by different 
algorithms. However, the patients classified by most of the clustering algorithms differ significantly in age at assessment, age of onset, obsession score, compulsion score, total score and depression. Clusters with more obsession, compulsion, total and depression scores contained patients with less age at assessment and less age of onset. No significant differences were detected between the two clusters for insight and avoidance levels. Clustering category-level raw data (Table 4) by most of the studied algorithms resulted in a cluster consisting of patients with more obsession, compulsion and total scores as well as more depression and avoidance levels in comparison to the other cluster. FCM, K-means and Ward+K-means algorithms detected more female to male and unoccupied to occupied ratios in the more severe group in comparison to the less severe one. Results of clustering category-based factor scores specified a patient cluster with less male to female and less occupied to unoccupied ratios that mostly were low educated people with more obsession, compulsion, total and depression scores and less insight level (Table 4). Other variables did not show significant differences between two clusters. Moreover, Complete algorithm produced two clusters that didn't have significant differences in most of the variables (Table 4).

\section{Discussion}

Researchers have suggested that OCD is actually composed of distinct subtypes which play important roles in its etiology and treatment. Studies have focused primarily on obsessive and compulsive symptom presentation to detect subtypes of OCD. In the present study we aimed to investigate the best model for symptom-based clustering of OCD patients based on YBOCS-CL which can be used for further elucidation of OCD pathophysiology and treatment. 
We applied five different algorithms (FCM, K-means, Ward, Ward + K-means and Complete) to detect the best fitted clustering method for grouping OCD patients. According to different scoring of YBOCS-CL we did not restrict initial data set to special type. We conducted clustering algorithms on raw Likert-scale item data and raw category data acquired based on the most common scoring strategy administered in previous studies (Baer, 1994; Calamari et al., 1999). Moreover, we conducted factor analysis for item and category level data and clustered patients based on their factor scores. To specify the best clustering model, we evaluated 2 to 6 clustering solutions to avoid missing the most appropriate model. There are no universal and effective criteria for selection of features and clustering schemes; so, we chose 4 commonly used clustering indices including Davies-Bouldin, Calinski-Harabasz, Silhouettes and Dunn indices to evaluate clustering models. Results revealed that OCD patients were not classified based on their symptoms but according to their symptoms severities. Several clustering studies published earlier used different measures and various clustering methods and evaluated the results by employing different criteria (Calamari et al., 1999; Calamari et al., 2004; Fals-Stewart and Lucente, 1993; Lochner et al., 2005; Lochner C, 2008). Three to seven OCD subgroups were identified in these studies with different characteristics. Differences in the identified dimensions or subgroups may have resulted from methodological inconsistencies in studies: different used analytic strategies, different OCD symptom measures, different methods for the YBOCS checklist quantification, psychometric limitations of quantifying the YBOCS checklist, variability in sample sizes and different criteria to evaluate clustering results. 
Results of the current study showed that almost all of the used clustering methods identified 2-cluster solution as the best fitted model and clustering raw data (item and category levels) resulted in models with better validity indices than clustering factor scores. It was interesting that none of the clustering algorithms was superior to the others. Moreover, Complete algorithm was the least appropriate method for clustering this sample. Comparing demographic and clinical characteristics of patients in two clusters revealed that they separate from each other based on their obsessive and compulsive severity. The more severe group had lower age at assessment and reported earlier age of onset. The correlation of severity and earlier age of onset was also reported previously (Kichuk et al., 2013). Moreover, category level data clustering revealed that severe group showed more female to male ratios, more unoccupied to occupied ratios, more low educated people, more depression and avoidance levels as well as less insight.

The limitation of the current study is relatively homogenous and mildly ill sample of patients (total mean severity \pm SD were $18.8 \pm 8.8$ ). Moreover, YBOCS severity scores in this study are current scores rather than worst-ever scores which may be a reflection of previous treatment effects. So the patients who reported severe symptoms were limited. However, findings of the current study are consistent with the previous LCA investigation on YBOCS-CL reported by Delucchi et al (Delucchi et al., 2011). The results of both investigations described OCD patients as a single spectrum based on severity or symptom endorsement rates. According to the results of these two studies heterogeneity of OCD patients based on the YBOCS symptoms which was recommended by the previous studies (Calamari et al., 1999; Lochner C, 2008) seems 
challenging and needs to be more studied. Attempts to classify OCD patients into subtypes have had limited success and were controversial. Although several studies searched for a correlation between OCD symptom subtypes and specific treatments, no precise match has been proposed yet (Starcevic and Brakoulias, 2008). However, our findings do not consistently argue against the existence of categorical subtypes of OCD. Possibly different measures must be used for different purposes in the assessment of OCD as mentioned previously by Anholt and colleagues (Anholt et al., 2009). Clustering OCD patients based on treatment course and response or comorbid characteristics rather than symptoms may produce more interpretable subgroups with clinical importance. Further investigations may clarify the most appropriate measures to assess complex heterogeneity of OCD patients for improved assessment and treatment of OCD patients.

\section{Acknowledgments}

We are grateful to Imam Hossain staff for their cooperation with our research team.

\section{Funding}

This research was supported by grants from the Iran National Science Foundation and Neuroscience Research Center, Shahid Beheshti University of Medical Sciences, Iran. 


\section{References}

Althoff, R.R., Rettew, D.C., Boomsma, D.I., Hudziak, J.J., 2009. Latent class analysis of the child behavior checklist obsessive-compulsive scale. Comprehensive psychiatry 50, 584-592. Anholt, G.E., van Oppen, P., Emmelkamp, P.M., Cath, D.C., Smit, J.H., van Dyck, R., van Balkom, A.J., 2009. Measuring obsessive-compulsive symptoms: Padua inventory-revised vs. Yale-Brown obsessive compulsive scale. Journal of Anxiety Disorders 23, 830-835.

Asadi, S., Daraeian, A., Rahmani, B., Kargari, A., Ahmadiani, A., Shams, J., 2016. Exploring yale-brown obsessive-compulsive scale symptom structure in Iranian OCD patients using itembased factor analysis. Psychiatry Research 245, 416-422.

Association, A.P., 1994. Diagnostic and statistical manual of mental disorders, 4 ed.

Baer, L., 1994. Factor analysis of symptom subtypes of obsessive compulsive disorder and their relation to personality and tic disorders. Journal of Clinical Psychiatry 55, 18-23.

Bezdek, J.C., Ehrlich, R., Full, W., 1984. FCM: The fuzzy c-means clustering algorithm.

Computers \& Geosciences 10, 191-203.

Bloch, M.H., Angeli Landeros-Weisenberger, M., Rosario, M.C., Pittenger, C., Leckman, J.F., 2008. Meta-analysis of the symptom structure of obsessive-compulsive disorder. The American journal of psychiatry 165, 1532-1542.

Calamari, J.E., Wiegartz, P.S., Janeck, A.S., 1999. Obsessive-compulsive disorder subgroups: a symptom-based clustering approach. Behaviour Research and Therapy 37, 113-125.

Calamari, J.E., Wiegartz, P.S., Riemann, B.C., Cohen, R.J., Greer, A., Jacobi, D.M., Jahn, S.C., Carmin, C., 2004. Obsessive-compulsive disorder subtypes: an attempted replication and extension of a symptom-based taxonomy. Behaviour Research and Therapy 42, 647-670. Caliński, T., Harabasz, J., 1974. A dendrite method for cluster analysis. Communications in Statistics-theory and Methods 3, 1-27.

Davies, D.L., Bouldin, D.W., 1979. A cluster separation measure. Pattern Analysis and Machine Intelligence, IEEE Transactions on, 224-227.

Defays, D., 1977. An efficient algorithm for a complete link method. The Computer Journal 20, 364-366.

Delucchi, K.L., Katerberg, H., Stewart, S.E., Denys, D.A., Lochner, C., Stack, D.E., den Boer, J.A., van Balkom, A.J., Jenike, M.A., Stein, D.J., 2011. Latent class analysis of the Yale-Brown Obsessive-Compulsive Scale symptoms in obsessive-compulsive disorder. Comprehensive psychiatry 52, 334-341.

Denys, D., de Geus, F., van Megen, H.J., Westenberg, H.G., 2004. Use of factor analysis to detect potential phenotypes in obsessive-compulsive disorder. Psychiatry research 128, 273280.

Fals-Stewart, W., Lucente, S., 1993. An MCMI cluster typology of obsessive-compulsives: A measure of personality characteristics and its relationship to treatment participation, compliance and outcome in behavior therapy. Journal of Psychiatric Research 27, 139-154.

Feinstein, S.B., Fallon, B.A., Petkova, E., Liebowitz, M.R., 2003. Item-by-item factor analysis of the Yale-Brown obsessive compulsive scale symptom checklist. The Journal of neuropsychiatry and clinical neurosciences 15, 187-193.

Goodman, W.K., Price, L.H., Rasmussen, S.A., Mazure, C., Fleischmann, R.L., Hill, C.L., Heninger, G.R., Charney, D.S., 1989. The Yale-Brown obsessive compulsive scale: I.

Development, use, and reliability. Archives of general psychiatry 46, 1006-1011.

Hasler, G., LaSalle-Ricci, V.H., Ronquillo, J.G., Crawley, S.A., Cochran, L.W., Kazuba, D., Greenberg, B.D., Murphy, D.L., 2005. Obsessive-compulsive disorder symptom dimensions show specific relationships to psychiatric comorbidity. Psychiatry research 135, 121-132. 
Ilc, N., 2012. Modified Dunn's cluster validity index based on graph theory. Przegląd Elektrotechniczny 88, 126-131.

Katerberg, H., Delucchi, K.L., Stewart, S.E., Lochner, C., Denys, D.A., Stack, D.E., Andresen, J.M., Grant, J., Kim, S.W., Williams, K.A., 2010. Symptom dimensions in OCD: item-level factor analysis and heritability estimates. Behavior genetics 40, 505-517.

Kichuk, S.A., Torres, A.R., Fontenelle, L.F., Rosário, M.C., Shavitt, R.G., Miguel, E.C., Pittenger, C., Bloch, M.H., 2013. Symptom dimensions are associated with age of onset and clinical course of obsessive-compulsive disorder. Progress in Neuro-Psychopharmacology and Biological Psychiatry 44, 233-239.

Lochner, C., Hemmings, S.M., Kinnear, C.J., Niehaus, D.J., Nel, D.G., Corfield, V.A., MoolmanSmook, J.C., Seedat, S., Stein, D.J., 2005. Cluster analysis of obsessive-compulsive spectrum disorders in patients with obsessive-compulsive disorder: clinical and genetic correlates.

Comprehensive psychiatry 46, 14-19.

Lochner C, H.S., Kinnear CJ, Nel D, Hemmings SM, Seedat S, Moolman-Smook JC, Stein DJ., 2008. Cluster analysis of obsessive-compulsive symptomatology: identifying obsessivecompulsive disorder subtypes. The Israel journal of psychiatry and related sciences 45, 164. Lochner, C., Stein, D.J., 2003. Heterogeneity of obsessive-compulsive disorder: a literature review. Harvard review of psychiatry 11, 113-132.

MacQueen, J., 1967. Some methods for classification and analysis of multivariate observations, Proceedings of the fifth Berkeley symposium on mathematical statistics and probability.

Oakland, CA, USA., pp. 281-297.

Mataix-Cols, D., do Rosario-Campos, M.C., Leckman, J.F., 2005. A multidimensional model of obsessive-compulsive disorder. American Journal of Psychiatry 162, 228-238.

Mataix-Cols, D., Baer, L., Rauch, S.L., Jenike, M.A., 2000. Relation of factor-analyzed symptom dimensions of obsessive-compulsive disorder to personality disorders. Acta Psychiatrica Scandinavica 102, 199-202.

McKay, D., Abramowitz, J.S., Calamari, J.E., Kyrios, M., Radomsky, A., Sookman, D., Taylor, S., Wilhelm, S., 2004. A critical evaluation of obsessive-compulsive disorder subtypes:

symptoms versus mechanisms. Clinical psychology review 24, 283-313.

Nestadt, G., Addington, A., Samuels, J., Liang, K.-Y., Bienvenu, O.J., Riddle, M., Grados, M., Hoehn-Saric, R., Cullen, B., 2003. The identification of OCD-related subgroups based on comorbidity. Biological Psychiatry 53, 914-920.

Nestadt, G., Di, C., Riddle, M., Grados, M., Greenberg, B., Fyer, A., McCracken, J., Rauch, S., Murphy, D., Rasmussen, S., 2009. Obsessive-compulsive disorder: subclassification based on co-morbidity. Psychological medicine 39, 1491-1501.

Noshirvani, H.F., Kasvikis, Y., Marks, I.M., Tsakiris, F., Monteiro, W., 1991. Gender-divergent aetiological factors in obsessive-compulsive disorder. The British Journal of Psychiatry 158, 260-263.

Rajezi Esfahani, S., Motaghipour, Y., Kamkari, K., Zahiredin, A., Janbozorgi, M., 2012.

Reliability and Validity of the Persian version of the Yale-Brown Obsessive-Compulsive scale (Y-BOCS). Iranian Journal of Psychiatry and clinical psychology 17, 297-303.

Rousseeuw, P.J., 1987. Silhouettes: a graphical aid to the interpretation and validation of cluster analysis. Journal of computational and applied mathematics 20, 53-65.

Starcevic, V., Brakoulias, V., 2008. Symptom subtypes of obsessive-compulsive disorder: are they relevant for treatment? Australian and New Zealand Journal of Psychiatry 42, 651-661.

Thomsen, P.H., Jensen, J., 1991. Latent class analysis of organic aspects of obsessive-compulsive disorder in children and adolescents. Acta Psychiatrica Scandinavica 84, 391-395.

Vermunt, J.K., Magidson, J., 2004. Latent class analysis. The sage encyclopedia of social sciences research methods, 549-553. 


\section{Table and Figure legends:}

Table 1: Demographic and clinical characteristics of OCD patients $(N=216)$

Table 2: Underlying factors specified from item-based and category-based factor analysis of YBOCS-CL.

Table 3: Clinical and demographic characteristics of patients separated by clustering the YBOCS-CL item-level raw data and item-based factor scores.

Table 4: Clinical and demographic characteristics of patients separated by clustering the YBOCS-CL category-level raw data and category-based factor scores.

Figure1: Evaluation of 2 to 6 cluster solutions resulted from conducting five clustering algorithms (FCM, K-means, Ward, Ward+K-means, Complete) on different sets of data by clustering indices. $\mathbf{A}_{1}-\mathbf{A}_{4}$ : Davies-Bouldin values of resulted models from applying clustering algorithms on item-level raw data, item-based factor scores, category-level raw data and category-based factor scores, respectively. $\mathbf{B}_{1}-\mathbf{B}_{4}$ : Calinski-Harabasz values of resulted models from applying clustering algorithms on item-level raw data, item-based factor scores, categorylevel raw data and category-based factor scores, respectively. $\mathbf{C}_{1}-\mathbf{C}_{4}$ : Silhouettes values of resulted models from applying clustering algorithms on item-level raw data, item-based factor scores, category-level raw data and category-based factor scores, respectively. $\mathbf{D}_{\mathbf{1}}-\mathbf{D}_{\mathbf{4}}$ : Dunn values of resulted models from applying clustering algorithms on item-level raw data, itembased factor scores, category-level raw data and category-based factor scores, respectively.

Figure2: Mean of symptom scores in patients separated by 2-solution clustering models resulted from conducting five clustering algorithms (FCM, K-means, Ward, Ward+K-means, Complete) on different sets of data. A1: Mean scores on the YBOCS-CL obsession and compulsion categories for each subgroup identified from clustering item-level raw data. A2: 
Mean YBOCS-CL item-based factor scores for each subgroup identified from clustering itembased factor scores. B1: Mean scores on the YBOCS-CL obsession and compulsion categories for each subgroup identified from clustering category-level raw data. B2: Mean YBOCS-CL category-based factor scores for each subgroup identified from clustering category-based factor scores. 
A1 FCM

$2_{0}^{4} \frac{4}{1}$

$$
1 \quad \text { K-means }
$$

2 2 म

Ward

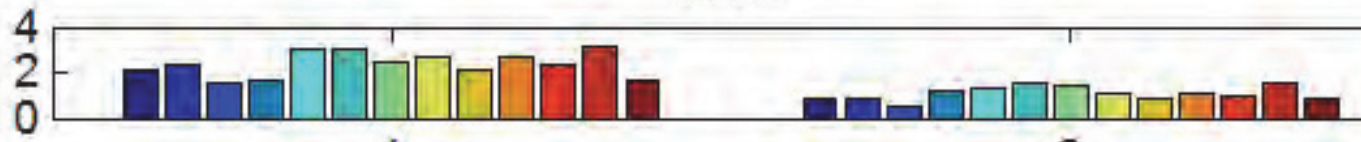

$$
1
$$

Ward+K-means

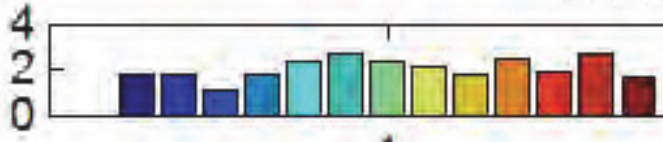$$
1
$$

Complete

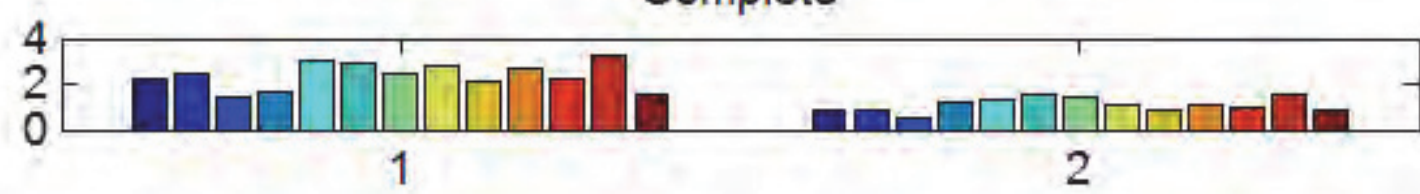

\section{Category-level raw data}

B1

FCM

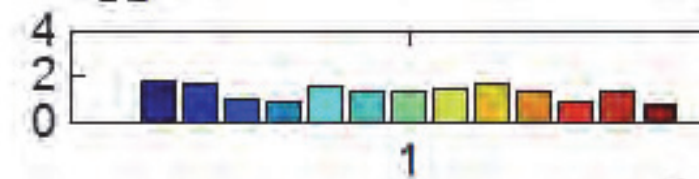

K-means

$$
2
$$
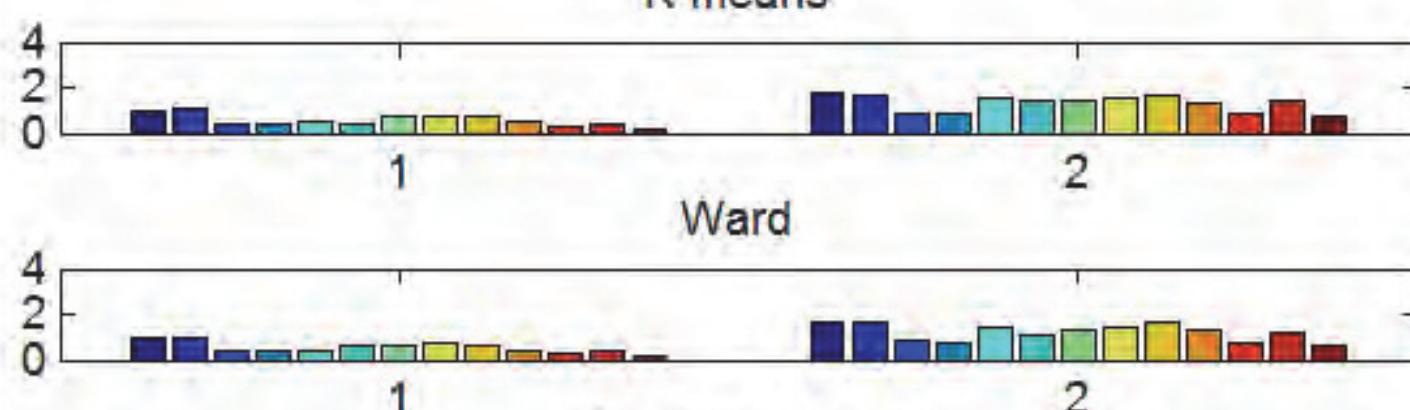

$$
1 \quad \text { Ward+K-means }
$$

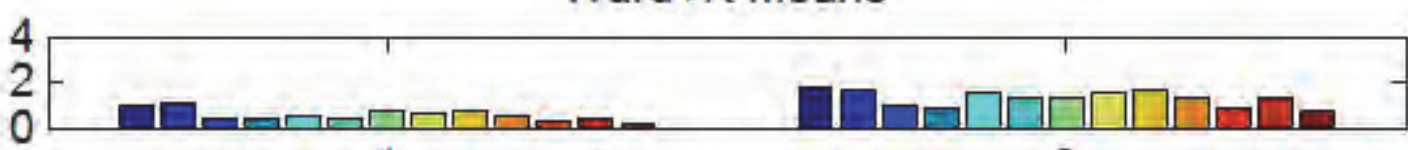

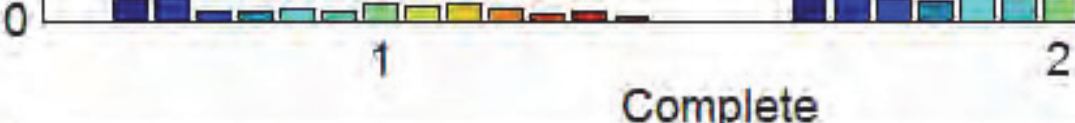

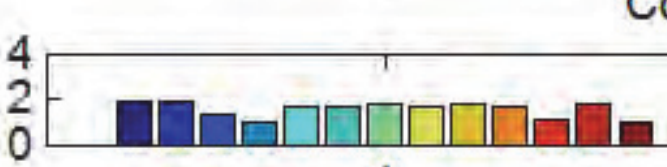

1

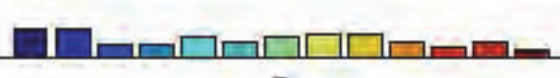

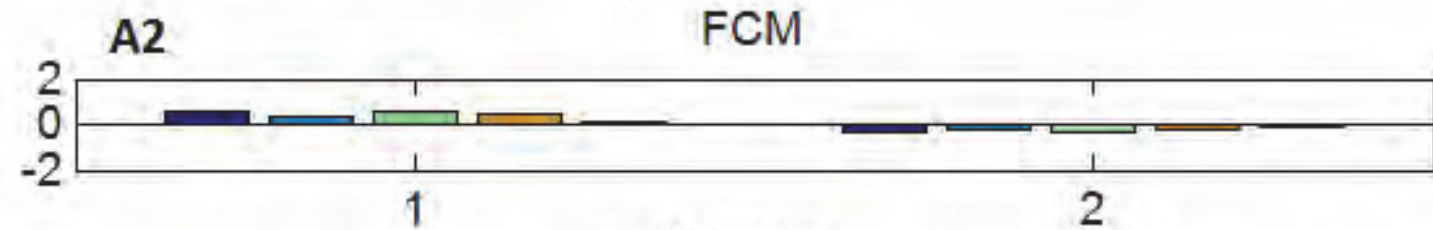

K-means
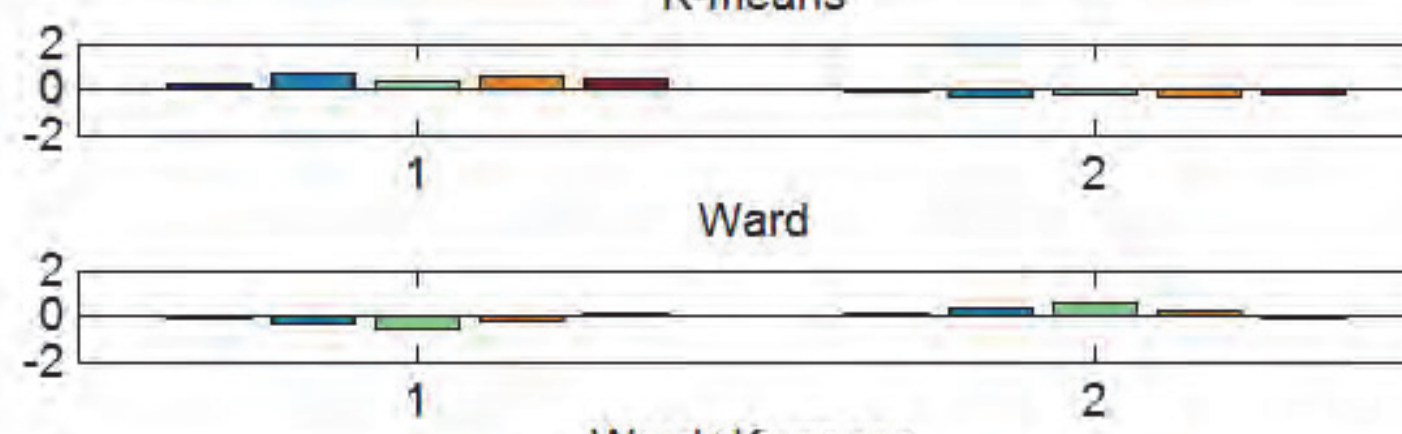

Ward +K-means

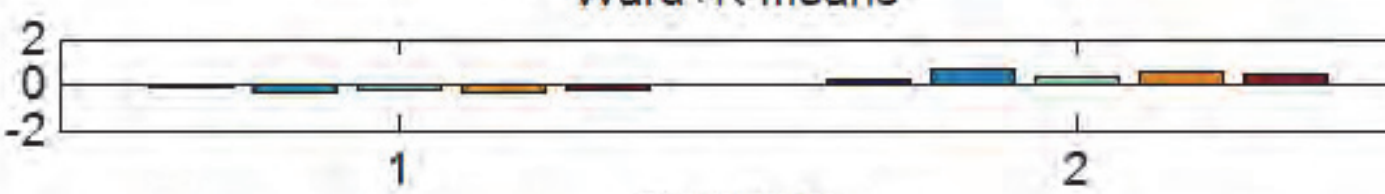

Complete

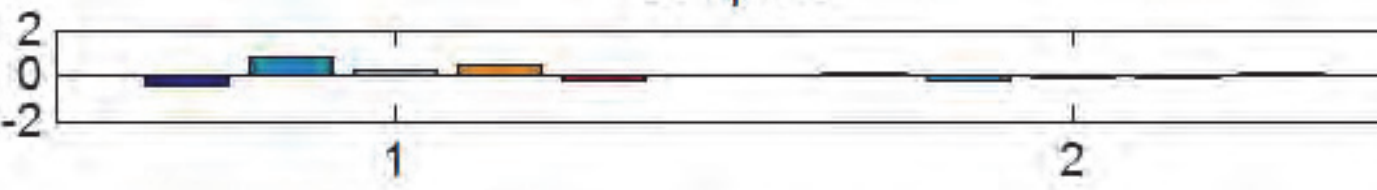

Category -based factor scores

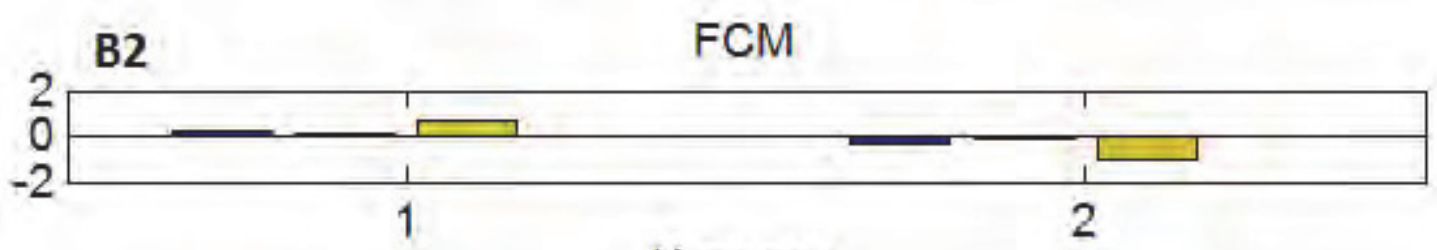

K-means

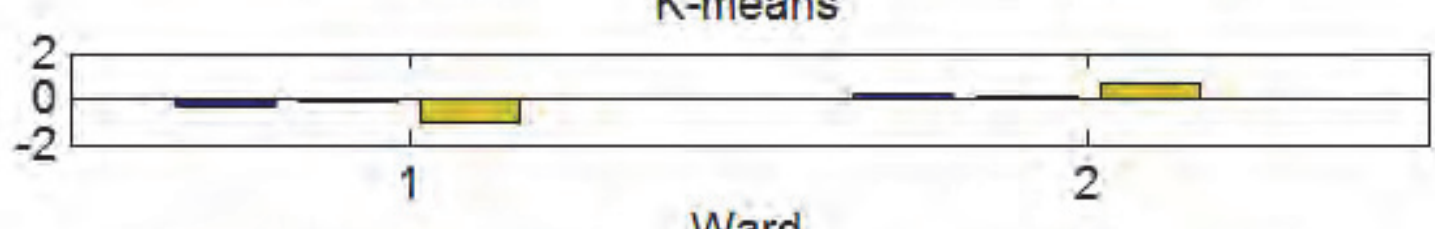

Ward

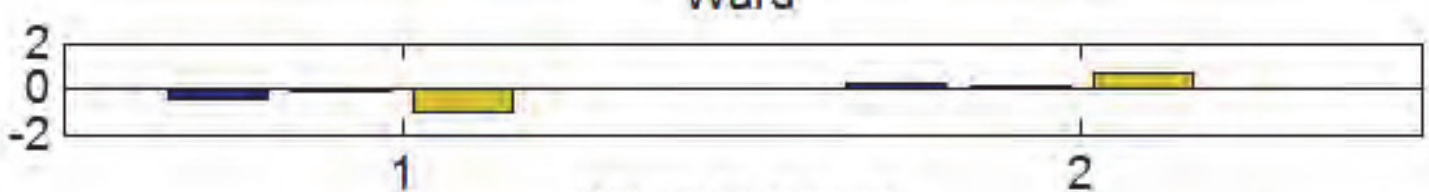

Ward +K-means

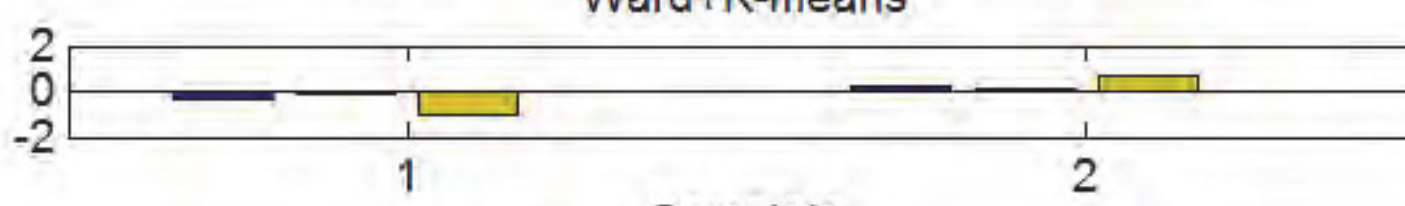

Complete

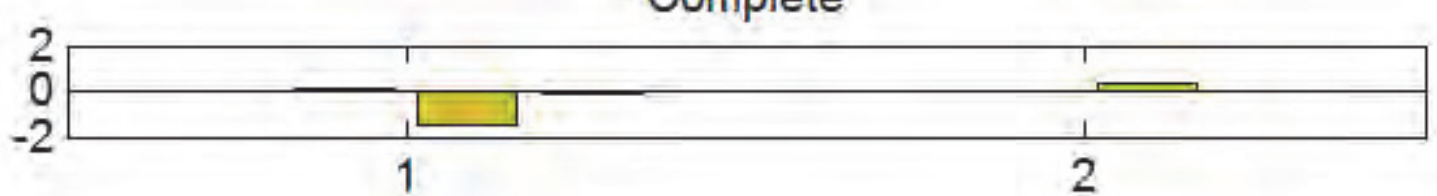

N $\mathrm{N}$

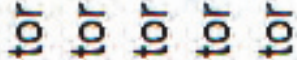

(ब

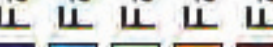

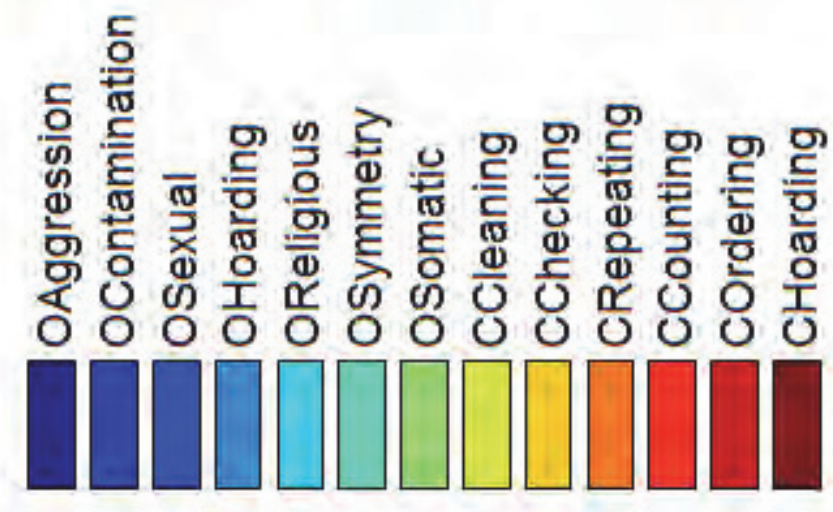


Table 1: Demographic and clinical characteristics of $O C D$ patients $(\mathrm{N}=216)$

\begin{tabular}{|c|c|c|c|}
\hline & & Number & $\%$ \\
\hline \multirow[t]{2}{*}{ Gender } & Male & 70 & 32 \\
\hline & Female & 146 & 68 \\
\hline \multirow[t]{2}{*}{ Marital status } & Single & 70 & 32 \\
\hline & Married & 145 & 68 \\
\hline \multirow[t]{4}{*}{ Educational level } & School dropout & 69 & 33 \\
\hline & Diploma & 93 & 43 \\
\hline & Under graduate & 44 & 20 \\
\hline & Graduate & 9 & 4 \\
\hline \multirow[t]{2}{*}{ Occupation } & Unemployed & 118 & 55 \\
\hline & Employed & 97 & 45 \\
\hline \multirow[t]{4}{*}{ Familial history of psychiatric disorders } & OCD & 101 & 47 \\
\hline & Other Axis I disorders & 59 & 27 \\
\hline & No history & 54 & 26 \\
\hline & & Mean & SD \\
\hline Age at assessment & & 33.7 & 10.2 \\
\hline Age of onset & & 23.7 & 10.7 \\
\hline Obsession Severity & & 10.3 & 4.7 \\
\hline Compulsion Severity & & 8.9 & 5.9 \\
\hline Total Severity & & 18.6 & 8.8 \\
\hline
\end{tabular}


Table 2: Underlying factors specified from item-based and category-based factor analysis of YBOCS-CL.

\begin{tabular}{cll}
\hline Factors & \multicolumn{1}{|c}{ Item-based } & \multicolumn{1}{c}{ Category-based } \\
\hline 1 & Aggression, Checking & $\begin{array}{l}\text { Aggression, Contamination, Sexual, Religious, Somatic, Checking, } \\
\text { Repeating }\end{array}$ \\
$\mathbf{2}$ & Contamination, Cleaning & Symmetry, Cleaning, Counting, Ordering \\
$\mathbf{3}$ & $\begin{array}{l}\text { Symmetry, Ordering, Counting, Repeating, } \\
\text { Hoarding }\end{array}$ & Contamination, Cleaning \\
$\mathbf{4}$ & Sexual & Hoarding \\
$\mathbf{5}$ & Somatic & \\
\hline
\end{tabular}


Table 3: Clinical and demographic characteristics of patients separated by clustering the YBOCS-CL item-level raw data and item-based factor scores.

\begin{tabular}{|c|c|c|c|c|c|c|c|c|c|c|c|c|c|c|c|c|c|c|c|c|c|}
\hline \multicolumn{12}{|c|}{ Item-level raw data } & \multicolumn{10}{|c|}{ Item-based factor scores } \\
\hline & \multirow[b]{2}{*}{ Cluster } & \multicolumn{2}{|c|}{ FCM } & \multicolumn{2}{|c|}{ K-means } & \multicolumn{2}{|c|}{ Ward } & \multicolumn{2}{|c|}{$\begin{array}{l}\text { Ward+ } \\
\text { K-means }\end{array}$} & \multicolumn{2}{|c|}{ Complete } & \multicolumn{2}{|c|}{ FCM } & \multicolumn{2}{|c|}{ K-means } & \multicolumn{2}{|c|}{ Ward } & \multicolumn{2}{|c|}{$\begin{array}{l}\text { Ward+ } \\
\text { K-means }\end{array}$} & \multicolumn{2}{|c|}{ Complete } \\
\hline & & 1 & 2 & 1 & 2 & 1 & 2 & 1 & 2 & 1 & 2 & 1 & 2 & 1 & 2 & 1 & 2 & 1 & 2 & 1 & 2 \\
\hline Age at & Mean & 34.9 & 31.9 & 32 & 34 & 29.4 & 34.3 & 32.1 & 34.4 & 29.1 & 34.3 & 31.9 & 34.6 & 30.8 & 35.1 & 35.1 & 32.2 & 35.1 & 30.8 & 32.9 & 34 \\
\hline assessment & P-value & \multicolumn{2}{|c|}{$\underline{0.03}$} & \multicolumn{2}{|c|}{0.1} & \multicolumn{2}{|c|}{$\underline{0.01}$} & \multicolumn{2}{|c|}{0.1} & \multicolumn{2}{|c|}{$\underline{0.01}$} & \multicolumn{2}{|c|}{0.06} & \multicolumn{2}{|c|}{$\leq 0.01$} & \multicolumn{2}{|c|}{$\underline{0.03}$} & \multicolumn{2}{|c|}{$\leq 0.01$} & \multicolumn{2}{|c|}{0.4} \\
\hline Age of & Mean & 25.6 & 21 & 21 & 25.4 & 20.3 & 24.2 & 20.6 & 25.1 & 20 & 24.2 & 20.6 & 25.4 & 20.3 & 25.4 & 26.3 & 21 & 25.4 & 20.3 & 22.7 & 24 \\
\hline onset & P-value & \multicolumn{2}{|c|}{$\leq 0.01$} & \multicolumn{2}{|c|}{$\leq 0.01$} & \multicolumn{2}{|c|}{0.07} & \multicolumn{2}{|c|}{$\leq 0.01$} & & & & 01 & & 01 & & & & 01 & & 3 \\
\hline Obsession & Mean & 9.23 & 12 & 12 & 9.3 & 12.5 & 10 & 12.3 & 9.5 & 12.5 & 10.1 & 11.9 & 9.5 & 11.9 & 9.6 & 9.6 & 11.2 & 9.6 & 11.9 & 11.1 & 10 \\
\hline score & P-value & & & & & & & & $\underline{01}$ & & & & & & 01 & & & & 01 & & \\
\hline Compulsion & Mean & 7.6 & 10.8 & 11 & 7.6 & 12.1 & 8.5 & 11.4 & 7.8 & 12.1 & 8.5 & 10.8 & 7.9 & 10.2 & 8.3 & 7.4 & 10.6 & 8.3 & 10.2 & 10.5 & 8.4 \\
\hline score & P-value & & & & & & & & & & & & & & 22 & & & & & & 2 \\
\hline Depression & Mean & 1.42 & 2.07 & 2.08 & 1.4 & 2.2 & 1.6 & 2.2 & 1.4 & 2.33 & 1.6 & 2.1 & 1.4 & 2.1 & 1.4 & 1.5 & 1.8 & 1.4 & 2.1 & 2 & 1.5 \\
\hline & P-value & & & & & & & & & & & & & & & & & & & & $\underline{3}$ \\
\hline Insight & Lack & 34 & 25 & 25 & 34 & 10 & 49 & 22 & 37 & 10 & 49 & 24 & 35 & 21 & 38 & 26 & 33 & 38 & 21 & 17 & 42 \\
\hline & Presence & 93 & 63 & 59 & 97 & 18 & 138 & 44 & 112 & 16 & 140 & 51 & 105 & 49 & 107 & 86 & 70 & 107 & 49 & 37 & 119 \\
\hline & P-value & & & & & & & & & & & & & & & & & & & & \\
\hline Avoidance & Lack & 85 & 49 & 46 & 88 & 16 & 118 & 36 & 98 & 14 & 120 & 42 & 92 & 38 & 96 & 73 & 61 & 96 & 38 & 30 & 104 \\
\hline & Presence & 42 & 39 & 38 & 43 & 12 & 69 & 30 & 51 & 12 & 69 & 33 & 48 & 32 & 49 & 39 & 42 & 49 & 32 & 24 & 57 \\
\hline & P-value & & & & & & & & 1 & & & & & & & & & & & & \\
\hline Gender & Female & 86 & 60 & 57 & 89 & 19 & 127 & 45 & 101 & 18 & 128 & 50 & 96 & 42 & 104 & 74 & 72 & 104 & 42 & 32 & 114 \\
\hline & Male & 41 & 28 & 27 & 42 & 9 & 60 & 21 & 48 & 8 & 61 & 25 & 44 & 28 & 41 & 38 & 31 & 41 & 28 & 22 & 47 \\
\hline & P-value & & & & & & & & 4 & & & & & & & & & & & & \\
\hline Job & Unemployed & 65 & 53 & 53 & 65 & 18 & 100 & 41 & 77 & 17 & 101 & 49 & 69 & 37 & 81 & 57 & 61 & 81 & 37 & 29 & 89 \\
\hline
\end{tabular}




\begin{tabular}{|c|c|c|c|c|c|c|c|c|c|c|c|c|c|c|c|c|c|c|c|c|c|}
\hline \multirow[b]{3}{*}{ History } & Employed & 62 & 35 & 31 & 66 & 10 & 87 & 25 & 72 & 9 & 88 & 26 & 71 & 33 & 64 & 55 & 42 & 64 & 33 & 25 & 72 \\
\hline & P-value & \multicolumn{2}{|c|}{0.18} & \multicolumn{2}{|c|}{$\underline{0.05}$} & \multicolumn{2}{|c|}{0.28} & \multicolumn{2}{|c|}{0.15} & \multicolumn{2}{|c|}{0.25} & \multicolumn{2}{|c|}{0.02} & \multicolumn{2}{|c|}{0.67} & \multicolumn{2}{|c|}{0.22} & \multicolumn{2}{|c|}{0.67} & \multicolumn{2}{|c|}{0.84} \\
\hline & No history & 35 & 19 & 18 & 36 & 7 & 47 & 15 & 39 & 7 & 47 & 16 & 38 & 16 & 38 & 34 & 20 & 38 & 16 & 15 & 39 \\
\hline & Axis I Dis & 30 & 29 & 26 & 33 & 9 & 50 & 18 & 41 & 8 & 51 & 22 & 37 & 22 & 37 & 28 & 31 & 37 & 22 & 15 & 44 \\
\hline & $O C D$ & 62 & 39 & 39 & 62 & 12 & 89 & 32 & 69 & 11 & 90 & 36 & 65 & 31 & 70 & 50 & 51 & 70 & 31 & 24 & 77 \\
\hline & P-value & \multicolumn{2}{|c|}{0.27} & \multicolumn{2}{|c|}{0.5} & \multicolumn{2}{|c|}{0.82} & \multicolumn{2}{|c|}{0.88} & \multicolumn{2}{|c|}{0.86} & \multicolumn{2}{|c|}{0.66} & \multicolumn{2}{|c|}{0.61} & \multicolumn{2}{|c|}{0.18} & \multicolumn{2}{|c|}{0.61} & \multicolumn{2}{|c|}{0.85} \\
\hline \multirow[t]{3}{*}{ Education } & Low & 93 & 69 & 68 & 94 & 23 & 139 & 54 & 108 & 21 & 141 & 64 & 98 & 54 & 108 & 85 & 77 & 108 & 54 & 38 & 124 \\
\hline & High & 34 & 19 & 16 & 37 & 5 & 48 & 12 & 41 & 5 & 48 & 11 & 42 & 16 & 37 & 27 & 26 & 37 & 16 & 16 & 37 \\
\hline & P-value & \multicolumn{2}{|c|}{0.38} & \multicolumn{2}{|c|}{0.12} & \multicolumn{2}{|c|}{0.37} & \multicolumn{2}{|c|}{0.14} & \multicolumn{2}{|c|}{0.49} & \multicolumn{2}{|c|}{0.01} & \multicolumn{2}{|c|}{0.67} & \multicolumn{2}{|c|}{0.84} & \multicolumn{2}{|c|}{0.67} & \multicolumn{2}{|c|}{0.32} \\
\hline Marital status & Single & 34 & 35 & 32 & 37 & 11 & 58 & 26 & 43 & 10 & 59 & 29 & 40 & 29 & 40 & 34 & 35 & 40 & 29 & 19 & 50 \\
\hline & Married & 93 & 53 & 52 & 94 & 17 & 129 & 40 & 106 & 16 & 130 & 46 & 100 & 41 & 105 & 78 & 68 & 105 & 41 & 35 & 111 \\
\hline & P-value & & & & & & & & & & & & & & & & & & & & \\
\hline
\end{tabular}

Table 4: Clinical and demographic characteristics of patients separated by clustering the YBOCS-CL category-level raw data and category-based factor scores.

\begin{tabular}{|c|c|c|c|c|c|c|c|c|c|c|c|c|c|c|c|c|c|c|c|c|c|}
\hline \multicolumn{12}{|c|}{ Category-level raw data } & \multicolumn{10}{|c|}{ Category-based factor scores } \\
\hline & & \multicolumn{2}{|c|}{$\mathrm{FCM}$} & \multicolumn{2}{|c|}{ K-means } & \multicolumn{2}{|c|}{ Ward } & \multicolumn{2}{|c|}{$\begin{array}{l}\text { Ward+ } \\
\text { K-means }\end{array}$} & \multicolumn{2}{|c|}{ Complete } & \multicolumn{2}{|c|}{ FCM } & \multicolumn{2}{|c|}{ K-means } & \multicolumn{2}{|c|}{ Ward } & \multicolumn{2}{|c|}{$\begin{array}{l}\text { Ward+ } \\
\text { K-means }\end{array}$} & \multicolumn{2}{|c|}{ Complete } \\
\hline & Cluster & 1 & 2 & 1 & 2 & 1 & 2 & 1 & 2 & 1 & 2 & 1 & 2 & 1 & 2 & 1 & 2 & 1 & 2 & 1 & 2 \\
\hline Age at & Mean & 35.6 & 31.6 & 35.5 & 31.5 & 35.4 & 32.3 & 35.1 & 31.7 & 29.4 & 34.7 & 33 & 34.6 & 34.5 & 33.1 & 34.5 & 33.2 & 34.5 & 33.1 & 33.2 & 33.8 \\
\hline assessment & P-value & \multicolumn{2}{|c|}{$\leq 0.01$} & \multicolumn{2}{|c|}{$\leq 0.01$} & \multicolumn{2}{|c|}{$\underline{0.02}$} & \multicolumn{2}{|c|}{$\leq 0.01$} & \multicolumn{2}{|c|}{$\leq 0.01$} & \multicolumn{2}{|c|}{0.2} & \multicolumn{2}{|c|}{0.3} & \multicolumn{2}{|c|}{0.36} & \multicolumn{2}{|c|}{0.3} & \multicolumn{2}{|c|}{0.6} \\
\hline Age of & Mean & 26.4 & 20.8 & 26.3 & 20.6 & 26.6 & 21.3 & 26.4 & 20.7 & 20.1 & 24.6 & 22.8 & 25.1 & 25.1 & 22.8 & 25.4 & 22.7 & 25.1 & 22.8 & 22.4 & 24.1 \\
\hline onset & P-value & \multicolumn{2}{|c|}{$\leq 0.01$} & \multicolumn{2}{|c|}{$\leq 0.01$} & \multicolumn{2}{|c|}{$\leq 0.01$} & \multicolumn{2}{|c|}{$\leq 0.01$} & \multicolumn{2}{|c|}{$\underline{0.01}$} & \multicolumn{2}{|c|}{0.1} & \multicolumn{2}{|c|}{0.1} & \multicolumn{2}{|c|}{0.08} & \multicolumn{2}{|c|}{0.1} & \multicolumn{2}{|c|}{0.3} \\
\hline Obsession & Mean & 9.4 & 11.4 & 9.4 & 11.5 & 9 & 11.5 & 9.4 & 11.4 & 12.5 & 9.8 & 11.4 & 8.9 & 9 & 11.4 & 8.6 & 11.5 & 9 & 11.4 & 9.7 & 10.5 \\
\hline score & P-value & \multicolumn{2}{|c|}{$\leq 0.01$} & \multicolumn{2}{|c|}{$\leq 0.01$} & & 01 & $\leq 0$ & .01 & & .01 & $\leq 0$ & & $\leq \underline{0}$ & & & & $\leq 0$. & .01 & 0. & \\
\hline
\end{tabular}




\begin{tabular}{|c|c|c|c|c|c|c|c|c|c|c|c|c|c|c|c|c|c|c|c|c|c|}
\hline Compulsion & Mean & 7.2 & 10.8 & 7.1 & 11.1 & 7.5 & 10.1 & 7.1 & 10.9 & 12.1 & 8.2 & 9.8 & 7.7 & 7.8 & 9.8 & 7.5 & 9.8 & 7.8 & 9.8 & 8.5 & 9 \\
\hline score & P-value & \multicolumn{2}{|c|}{$\leq 0.01$} & \multicolumn{2}{|c|}{$\leq 0.01$} & \multicolumn{2}{|c|}{$\leq 0.01$} & \multicolumn{2}{|c|}{$\leq 0.01$} & \multicolumn{2}{|c|}{$\leq 0.01$} & \multicolumn{2}{|c|}{$\underline{0.01}$} & \multicolumn{2}{|c|}{$\underline{0.01}$} & \multicolumn{2}{|c|}{$\leq 0.01$} & \multicolumn{2}{|c|}{$\underline{0.01}$} & \multicolumn{2}{|c|}{0.5} \\
\hline \multirow[t]{2}{*}{ Depression } & Mean & 1.39 & 2 & 1.4 & 2 & 1.4 & 1.9 & 1.38 & 2 & 2.2 & 1.5 & 1.96 & 1.3 & 1.3 & 1.9 & 1.34 & 1.9 & 1.3 & 1.9 & 1.3 & 1.7 \\
\hline & P-value & \multicolumn{2}{|c|}{$\leq 0.01$} & \multicolumn{2}{|c|}{$\leq 0.01$} & \multicolumn{2}{|c|}{$\underline{0.01}$} & \multicolumn{2}{|c|}{$\leq 0.01$} & \multicolumn{2}{|c|}{$\leq 0.01$} & \multicolumn{2}{|c|}{$\leq 0.01$} & \multicolumn{2}{|c|}{$\leq 0.01$} & \multicolumn{2}{|c|}{$\leq 0.01$} & \multicolumn{2}{|c|}{$\leq 0.01$} & \multicolumn{2}{|c|}{0.08} \\
\hline \multirow[t]{3}{*}{ Insight } & Lack & 27 & 32 & 28 & 31 & 25 & 34 & 27 & 32 & 14 & 45 & 43 & 16 & 17 & 42 & 16 & 43 & 17 & 42 & 11 & 48 \\
\hline & Presence & 85 & 71 & 90 & 66 & 75 & 81 & 87 & 69 & 28 & 128 & 83 & 73 & 73 & 83 & 65 & 91 & 73 & 83 & 34 & 122 \\
\hline & P-value & \multicolumn{2}{|c|}{0.25} & \multicolumn{2}{|c|}{0.17} & \multicolumn{2}{|c|}{0.45} & \multicolumn{2}{|c|}{0.18} & & & & & & & & & & & & \\
\hline Avoidance & Lack & 77 & 57 & 81 & 53 & 72 & 62 & 78 & 56 & 22 & 112 & 74 & 60 & 60 & 74 & 55 & 79 & 60 & 74 & 28 & 106 \\
\hline & Presence & 35 & 46 & 37 & 44 & 28 & 53 & 36 & 45 & 20 & 61 & 52 & 29 & 30 & 51 & 26 & 55 & 30 & 51 & 17 & 64 \\
\hline & P-value & & & & & & & & & & & & & & & & & & & & \\
\hline Gender & Female & 68 & 78 & 72 & 74 & 64 & 82 & 70 & 76 & 30 & 116 & 97 & 49 & 50 & 96 & 46 & 100 & 50 & 96 & 23 & 123 \\
\hline & Male & 44 & 25 & 46 & 23 & 36 & 33 & 44 & 25 & 12 & 57 & 29 & 40 & 40 & 29 & 35 & 34 & 40 & 29 & 22 & 47 \\
\hline & P-value & & & & & & 25 & & & & & & & & & & & & & & 01 \\
\hline Job & Unemployed & 54 & 64 & 58 & 60 & 51 & 67 & 55 & 63 & 26 & 92 & 79 & 39 & 40 & 78 & 38 & 80 & 40 & 78 & 20 & 98 \\
\hline & Employed & 58 & 39 & 60 & 37 & 49 & 48 & 59 & 38 & 16 & 81 & 47 & 50 & 50 & 47 & 43 & 54 & 50 & 47 & 25 & 72 \\
\hline & P-value & & & & & & & & & & & & & & & & & & & & \\
\hline History & No History & 29 & 25 & 30 & 24 & 29 & 25 & 29 & 25 & 11 & 43 & 29 & 25 & 25 & 29 & 23 & 31 & 25 & 29 & 9 & 45 \\
\hline & Axis I Dis & 27 & 32 & 29 & 30 & 21 & 38 & 28 & 31 & 11 & 48 & 37 & 22 & 23 & 36 & 19 & 40 & 23 & 36 & 11 & 48 \\
\hline & $O C D$ & 56 & 45 & 58 & 43 & 49 & 52 & 56 & 45 & 20 & 81 & 59 & 42 & 42 & 59 & 39 & 62 & 42 & 59 & 25 & 76 \\
\hline & P-value & & & & & & & & & & & & & & & & & & & & \\
\hline Education & Low & 81 & 81 & 86 & 76 & 71 & 91 & 82 & 80 & 33 & 129 & 101 & 61 & 61 & 101 & 55 & 107 & 61 & 101 & 27 & 135 \\
\hline & High & 31 & 22 & 32 & 21 & 29 & 24 & 32 & 21 & 9 & 44 & 25 & 28 & 29 & 24 & 26 & 27 & 29 & 24 & 18 & 35 \\
\hline & P-value & & & & & & & & & & & & & & & & & & & & \\
\hline Marital status & Single & 31 & 38 & 32 & 37 & 26 & 43 & 31 & 38 & 17 & 52 & 40 & 29 & 30 & 39 & 27 & 42 & 30 & 39 & 16 & 53 \\
\hline
\end{tabular}


Married

$\begin{array}{llll}74 & 72 \quad 83\end{array}$

$63 \quad 25$ 121 